\title{
Information and discriminability as determinants of absolute judgment choice reaction time
}

MICHAEL E, DOHERTY

BOWLING GREEN STATE UNIVERSITY

Hick's law was generalized to the case of absolute judgment by measuring $S$ 's judgment time (JT) to unidimensionally varying, visual stimuli. The linear component of the regression of JT on transmitted information was highly significant, and was the only significant component, thus confirming Hick's law. Discriminability alone was found to be incapable of accounting for JT variance, although the results verified that, in the range manipulated, discriminability does affect performance. Further analyses revealed that stimulus-onset uncertainty does not, as Hick supposed, have the effect of adding one more state of the alternatives, and that Hick's law does not hold when applied to JTs to individual stimuli.

Application of Shannon's (1949) information measure to behavioral research has led to important generalizations about the human's "channel capacity" and also his "rate of gain" of information. Miller (1956) reviewed the absolute judgment (AJ) literature and concluded that the maximum number of unidimensionally varying stimuli that $S$ s can accurately identify is about seven. When $E$ requires $S$ to attempt to identify more than seven stimuli, S's error rate increases so as to stabllize the average amount of information transmitted, $T(S ; R$ ) (Attneave, 1959), at approximately 2.7 bits. Hick (1952) showed that "...the rate of gain of information is, on the average, constant with respect to time...". This generalization, which has come to be known as Hick's law, is based upon research in which Ss depressed one of a set of response keys when one of a set of stimulus lights was lit. The hypothesis of constancy of rate of gain was confirmed by the linearity of the regression of reaction time (RT) on information.

Assuming that stimulus-onset uncertainty (S-OU) added uncertainty to the stimulus display in an amount exactly equivalent to one more state of the stimulus set, Hick plotted RT against $\log (n+1)$ instead of $\log n$ (when $n$ is the number of stimulus alternatives). He supported this assumption in two ways: (1) with $n+1$, the best fitting linear functions extrapolated back through the origin, and (2) the $n+1$ expression consistently fit his data better.

Hick's experiment was not an AJ procedure, since all of the comparison stimuli, the unlit bulbs, were present on every trial. Furthermore, the dimensionality of the stimulus set he used is extremely difficult to specify. Stimuli varying along the "di- mension" of spatial position are clearly not unidimensional in Miller's sense since, if Ss were to count, $T(S ; R)$ would be limited only by the number of stimuli. Such is not the case with pitch, loudness, brightness, length of line, or any other dimension with which Miller dealt.

Whereas Ss in the studies reviewed by Miller were allowed virtually unlimited time to make AJs of unidimensional stimuli, Hick's Ss were pressed for time to respond to stimuli of unknown dimensionality. Replications of Hick's study have also used stimuli of unspecifiable dimensionality (Hyman, 1952; Fitts \& Switzer, 1962; Lamb \& Kaufman, 1965). The present research joins certain aspects of Miller's and Hick's paradigms by requiring $\mathrm{Ss}$ to make $\mathrm{AJ}$ of stimuli of known dimensionality as rapidly as they can. Thus, Hick's law will be tested for an AJ task with known dimensionality. Considering Crossman's (1955) original contention, later withdrawn (1964), that Hick's law was an artifact of the confounding of information with discriminability, or "confusability" (the C term in Crossman, 1955, Equation 9), an additional goal of the present research is to evaluate the effect of discriminability on choice time. Miller's generalization is already established (Miller, 1956) for the stimulus dimension employed, so no effort will be made to present enough stimulus information $(H(S))$ to measure S's channel capacity.

Specifically, the present research is aimed at testing three experimental hypotheses. (1) Hick's law-the amount of information which $S$ transmits in an AJ task is a linear function of JT. (2) Hick's additional assumption that S-OU adds uncertainty equivalent to one more state of the stimulus set. (3) Crossman's hypothesis that the increase in JT can be explained strictly on the basis of differences in confusability.

\section{METHOD}

\section{Subjects}

The Ss were 36 unpaid volunteers from E's classes in general and child psychology at the University of Connecticut. They were randomly assigned to six experimental conditions, with the restriction that each condition have six Ss.

\section{Apparatus}

A hand switch operated by $S$ presented visual 
stimuli in a Gerbrands Harvard tachistoscope and started a Hunter Klockounter. A verbal response activated a Gerbrands voice key, terminating stimulus presentation and stopping the Klockounter. Operation of the hand switch by $\mathrm{S}$ eliminated S-OU.

The stimuli were single, straight black lines of different lengths on plain white backgrounds. Appropriate differences in length were determined by preliminary psychophysical research. The stimuli were made by placing lengths of $3 / 16$ in. wide, black graph tape on 8-1/2 $x \quad 11$ in. oak tag cards. The center of the line was in the same place on each card, displaced slightly above the exact center of the card. The lines were in a geometric progression, the shortest being $1 \mathrm{in.,}$ the rest successive multiples of $1 \mathrm{in}$. by 1.05. Two stimulus sets were selected from this series. A low discriminabllity (HARD) set was made up of the first eight stimuli, with a range of from 1 in. to 1.41 in. The high discriminability (EASY) set was every fourth length, beginning with the first, the range for eight stimuli being from $1 \mathrm{in}$. to $3.92 \mathrm{in}$. For an average $S$, the adjacent stimuli differed from one another by about three DLs in the HARD set, and about $12 \mathrm{DLs}$ in the EASY set.

\section{Design}

The two discriminability levels were factorially combined with three $H(S)$ levels, yielding six experimental conditions. Discriminability was manipulated by having Ss respond to elther the HARD or EASY stimulus sets. The number of equiprobable Btimuli ( $n$ ) varied such that $n=2,4$, or 8 . For $n=2$, the stimull were the two shortest; for $n=4$, the four shortest. The $\mathrm{H}(\mathrm{S})$ was manipulated by having Ss respond to only one of the above alphabet sizes. Maximum $H(S)$ for the three conditions was 1,2 , and 3 bits per stimulus, for 2, 4, and 8 stimuli, respectively.

In order to maximize S-R compatibility (Fitts \& Deininger, 1954; Fitts \& Seeger, 1953) and minimize the influence of practice, the numbers 1 through 8 were selected as the responses, the shortest stimulus being named 1 , the next shortest, 2, etc. The numbers were pronounced 'bun, boo, bee, bore, bive, bix, bev, and bate" to minimize constant differences in roice key activation.

\section{Procedure}

First, E administered 15 simple reaction time (SRT) trials to Ss. These consisted of having Ss respond "bu" as quickly as they could to E's variably delayed tachistoscopic presentation of a blank stimulus field. Thus, SRT refers to RT as a function of S-OU. The $E$ then showed $S$ the stimuli appropriate to the condition to which $S$ had been assigned, naming the stimuli for $S$ and instructing $S$ to name the stimuli as he looked at them. Blocks of 25 ran- domized judgment trials were then administered until $S$ indicated unwillingness to continue. The Ss were generally administered 125 to 175 judgment trials, in only one case as few as 100. A judgment trial consisted of four steps: (1) E inserted the stimulus card and reset the Klockounter and voice key. The volce key reset button made an audible click, cueing $S$ that the apparatus was ready. (2) $S$ operated the hand switch which presented the stimulus. (3) $S$ verballzed the response as soon as he could. (4) E announced the correct response and S's judgment time (JT). After each block of trials, $S$ rested briefly. Preceding each block after the first, $S$ was given one practice trial, the stimulus for which was always the last stimulus of the preceding block.

\section{Infomational effects}

\section{RESULTS ANO DISCUSSION}

Each S's JT and number of errors per block were plotted for blocks of eight trials, and asymptotic performances were visually estimated from these plots. Mean JT $(\overline{\mathrm{JT}})$ and $\mathbf{T}(\mathrm{S} ; \mathrm{R})$ at asymptote were then calculated separately for each $S$ (see Table 1). For both discriminability conditions the linear component of the regression of JT on $T(S ; R)$ was statistically significant (EASY: $F=22.94, \mathrm{df}=1 / 14, \mathrm{p}<.001$; HARD: $F=19.49$, df $=1 / 14, p<.001$ ), while the quadratic and cubic components failed to attain acceptable levels of significance. Significant linear components, coupled with nonsignificant higher-order components, provide the strongest possible evidence for the generalization of Hick's law to AJs.

As further evidence for the dependence of RT on information, Hick has adduced positive correlations between $H_{1}(R)$, the residual uncertainty in the responses when the stimuli are given, and both $\mathrm{RT}_{\mathbf{1}}$ and $\mathbf{R T}_{j}$, the RTs associated with individual stimuli and responses, respectively. Hick implied that this relationship was similar to that between RT and $T(S ; R)$ and supported the constancy hypothesis, Hick's law. It does not. It violates Hick's law. The quantity $H_{i}(R)$ is not a measure of information extracted, but rather of information left over. The positive correlation between $H_{i}(R)$ and $R_{1}$ means that $\mathrm{Ss}$ were responding faster to those stimuli on which they were more frequently correct. The same relationship holds in the data of the present study. The correlations for most Ss are positive. Inspection of Hick's Table I and of the data of the present study reveals that the positive correlations are due primarily to the operation of an "end effect"; the Ss identified extreme stimuli both more accurately and more rapidly. Some function other than Hick's law, therefore, describes $\mathrm{Ss}^{\prime}$ behavior with respect to the individual stimuli.

Within the narrow range in which $H(S)$ was manipulated, $T(S ; R)$ varied directly with $H(S)$, the slopes 
Table 1. Values of individual $S^{\prime} \mathbf{s}$ mean judgment time $(\overline{\mathbf{J} T})$ in sec: and transmitted information $r(S ; R)$ in bits per stimulus.

\begin{tabular}{ccccc} 
& \multicolumn{4}{c}{ Discriminability Condition } \\
No. of Stimuli & $\mathrm{T}(\mathrm{S} ; \mathrm{R})$ & $\mathrm{JT}$ & $\mathrm{T}(\mathrm{S} ; \mathrm{R})$ & $\mathrm{JT}$ \\
\hline 2 & 0.0389 & .368 & 0.8851 & .330 \\
& 0.0640 & .416 & 0.5796 & .608 \\
& 0.1346 & .439 & 0.6825 & .387 \\
& 0.4370 & .625 & 0.7498 & .630 \\
& 0.0449 & .432 & 0.2059 & .440 \\
& 0.0031 & .464 & 0.6473 & .463 \\
& 0.5758 & .643 & 0.9542 & .545 \\
& 0.4537 & .841 & 1.5690 & .708 \\
& 0.6592 & .652 & 1.5066 & .878 \\
& 0.4476 & .874 & 0.8404 & .451 \\
& 0.6998 & .653 & 1.4543 & .673 \\
8 & 0.6949 & .751 & 1.5654 & .786 \\
& 0.7132 & .858 & 1.5763 & .912 \\
& 0.9751 & .977 & 1.8403 & .682 \\
& 1.422 & 1.119 & 2.0744 & .763 \\
& 1.5740 & .676 & 1.3025 & .660 \\
& 1.0289 & .625 & 1.6419 & .672 \\
& 1.0947 & .898 & 1.9645 & .857 \\
\hline
\end{tabular}

of the regressions of $T(S ; R)$ on $H(S)$ being virtually identical for the two discriminability conditions. It might be noted in this context that Es who disregard even a relatively small percentage of errors are introducing into their analyses a source of potentially serious bias. $S B^{\prime} T(S ; R)$ values in this study were markedly below their $H(S)$ values. The information loss is greatest with the first few errors, especially in the presence of a systematic response bias.

As has been clearly pointed out by Kornblum (1967), in a random series of equiprobable events, there is a confounding of information with the number of repetitions of events. Since the RT is shorter to repetitions than to alternations, or nonrepetitions (Bertelson, 1961, 1963, 1965, 1966; Kornblum, 1967), the confounding is crucial to the interpretation of Htck's law. Each S's data, therefore, was separated into repetitions and alternations, and $\overline{J T}$ and $T(S ; R)$ measures calculated separately for each. Removal of the repetitions from the data matrices ralsed the linear correlation between $T(S ; R)$ and $\overline{\mathrm{JT}}$ for the EASY condition from .72 to .89 , and lowered that for the HARD condition from .78 to .75. There were too few repetitions in the high information conditions to permit an overall analysis of the repetitions per se. Since, however, there was an approximately equal number of repetitions and nonrepetitions in the $n=2$ conditions, these data were examined in detail. The $\overline{\mathrm{JT}} 8$ were virtually identical for the HARD condition S8, the means differing by only $6 \mathrm{msec}$. The EASY $n=2 \mathrm{Ss}$, however, tended to be both faster $(t=1.36$, $d f=5, n s)$ and more accurate $(t=1.77, d f=5, n s)$ on the nonrepetitions. The $T(S ; R)$ values for the HARD Ss also tended to be higher for the nonrepetitions $(t=1.43, d f=5, n s)$. While these differences are not significant, they are in the opposite direction of what has previously been reported. To see if this "gambler's fallacy" effect was due to long runs of repetitions, the JTs of the EASY $n=2$ Ss were separated into alternations, first repetitions, second, third, fourth, and fifth and higher-order repetitions. In contrast with Bertelson $(1961,1966)$ there was a tendency for $J T$ to increase with repetition. The effect, however, was present on the first repetition. The repetition analysis cannot very well be carried out in more detail since this study was not designed to manipulate information and number of repetitions independently. The above results do show, nevertheless, that the informational effects are not an artifact of the confounding with repetition, although repetition is an important determinant of RT. The results are also of interest to anyone concerned with the repetition effect per se. Since they are the opposite of Bertelson's and Kornblum's findings, methodological differences may shed some light on the controlling variables. The two major differences seem to be the following. First, this study used a discrete task; with long response-signal intervals; theirs were self-paced with short intervals. Bertelson's $(1961,1966)$ studies showed that the repetition effect decreases as this interval increases up to 1 sec. Since, in the present study, the responsesignal interval was several seconds, it is quite possible that the repetition effect actually reverses itself over time, much as time-order error. Second, the AJ task in the present study is a radically different one from those employed by Bertelson and Kornblum.

\section{S-OU effects}

Hick's assertion that S-OU has the effect of adding one more stimulus alternative has been repeatedly challenged (Klemmer, 1957; Welford, 1962; Lamb \& Kaufman, 1965). This study provided further evidence against Hick's $n+1$ assumption. Hick's position demands that the extrapolation of the $\overline{\mathrm{JT}} / \mathrm{T}(\mathrm{S} ; \mathrm{R})$ regression intersect the ordinate at the one alternative, S-OU present point. This point is provided by the mean of the SRT data $(\overline{\mathrm{SRT}})$. For both the EASY and HARD conditions, $\overline{\mathrm{SRT}}=.272 \mathrm{sec}$, while the intercept constants were .335 and $.484 \mathrm{sec}$, respectively. The obtained (i.e., extrapolated) and theoretical (i.e., $\overline{S R T}$ ) intercept values were significantly different (EASY: $t=4.85, d f=35, p<.001$; HARD: $t=$ 16.30, $\mathrm{df}=35, \mathrm{p}<.001$ ). The data fail to support Hick's position.

Table 2. Values of Crossman's (1955) Confusability function (C)

\begin{tabular}{ccc} 
No. of Stimuli & \multicolumn{2}{c}{ Discriminability Condition } \\
Hard & Eosy \\
\hline 2 & 47.17 & 11.57 \\
4 & 102.65 & 25.15 \\
8 & 162.34 & 40.10 \\
\hline
\end{tabular}




\section{Discriminability effects}

Crossman's (1955) C function is a measure of discriminability which takes into account the number of stimuli in a set as well as the differences in stimulus magnitude. The value of $\mathrm{C}$ was calculated for each of the six sets of stimuli (see Table 2) and the $\mathrm{C}$ function was evaluated by plotting each $\mathrm{S}^{\prime} \mathrm{S}$ $\overline{\mathrm{JT}}$ against the value of $\mathrm{C}$ appropriate to S's condition. Separate regression lines were fitted to the EASY and HARD data, and the differences between the slopes tested. The regression coefficients were significantly different $(t=5.78, d f=32, p<.001)$. Discriminability differences as measured by the $\mathrm{C}$ function cannot alone, therefore, account for the relationship between $T(S ; R)$ and $\overline{J T}$, at least not in the discriminability range sampled. This is not to say that discriminability does not affect performance. Looking again at the relationship between $T(S ; R)$ and $\overline{\mathrm{JT}}$, this time with discriminability as a parameter there are no significant differences between the HARD and EASY conditions with respect to the slopes of the regressions of $\overline{J T}$ on $T(S ; R)(t=.87$, $\mathrm{df}=32, \mathrm{~ns})$ or of $\mathrm{T}(\mathrm{S} ; \mathrm{R})$ on $\overline{J T}(t=1.28, \mathrm{df}=32, \mathrm{~ns})$. Nor is there a difference between the HARD and EASY $\overline{\mathrm{JT} S}(\mathrm{t}=1.00, \mathrm{df}=32, \mathrm{~ns})$. A test of the difference between $\overline{\mathrm{JT} S}$ is not, however, an entirely satisfactory assessment of the influence of discriminability on $\overline{\mathrm{JT}}$, since the $\mathrm{Ss}$ in the two conditions transmitted radically different amounts of information. Testing the $\overline{\mathrm{JT}}$ differences equated for $\mathrm{T}(\mathrm{S} ; \mathrm{R})$ yields highly significant $t$ values throughout the $T(S ; R)$ range. Finally, the EASY condition Ss transmitted significantly more information than did the HARD condition Ss $(t=4.99, \mathrm{df}=32, \mathrm{p}<.001)$. The absence of statistically significant differences in the slopes, coupled with the differences in the levels of the functions, suggest that the two independent variables in this study, information and discriminability, affect $J T$ in an additive fashion.

\section{References}

Atneave, F. Applications of information theory to psychology. New York: Holt-Dryden, 1959.
Bertelson, P. Sequential redundancy and speed in a serial twochoice responding task, Quart. J. exp. Psychol., 1961, 13, 90-102.

Bertelson, P. S-R relationships and reaction times to new versus repeated signals in a serial task. J. exp. Psychol., 1963, 65, 478-484.

Bertelson, P. Serial choice reaction time as a function of response versus signal-and-response repetition. Nature, 1965, 206, 217218.

Bertelson, P., \& Renkin, A. Reaction times to new versus repeated signals in a serial task as a function of response-signal time interval. Acta Psychol., 1966, 25, 132-136.

Crossman, E. R. F. W. The measurement of discriminability. Quart. J. exp. Psychol., 1955, 7, 176-195.

Crossman, E. R. F. W. Reply to Dr. I. M. Hughes. Quart. J. exp. Psychol., 1964, 16, 181-183.

Fitts, P. M., \& Deininger, R. L. S-R compatibility: correspondence among paired elements within stimulus and response codes. $J$. exp. Psychol., 1954, 48, 483-492.

Fitts, P. M., \& Seeger, C. M. S-R compatibility: spatial characteristics of stimulus and response codes. J. exp. Psychol., $1953,46,199-210$.

Fitts, P. M., \& Switzer, C. Cognitive aspects of information processing: I. The familiarity of S-R sets and subsets. J. exp. Psychol., 1962, 63, 321-329.

Hyman, R. Stimulus information as a determinant of reaction time. J. exp. Psychol., 1953, 45, 188-196.

Klemmer, E. J. Simple reaction time as a function of time uncertainty. J. exp. Psychol., 1957, 54, 195-200.

Kornblum, S. Choice reaction time for repetitions and non-repetitions-a re-examination of the information hypothesis. Acta Psychol., 1967, 27, 178-187.

Lamb, J. C., \& Kaufman, H. Information transmission with unequally likely alternatives. Percept. mot. Skills, 1965, 21, 255-259.

Miller, G. A. The magical number seven, plus or minus two: some limits on our capacity for processing information. Psychol. Rev.. $1956,63,81-97$.

Welford, A. T. The measurement of sensory-motor performance: Survey and reappraisal of twelve years' progress. Ergonomics, $1960,3,189-229$.

\section{Note}

1. This article is based upon a dissertation submitted to the Department of Psychology at the University of Connecticut, in partial fulfillment of the requirements for the Ph.D. degree. The author is indebted to H. M. Kaufman, who directed the research. The computational part of this work was carried out by the Computer Center of the University of Connecticut, which is supported in part by Grant GP-1819 of the National Science Foundation.

(Accepted for publication August 25, 1967.) 